\title{
Constructing City Ontology from Expert for Smart City
}

\section{Management}

\author{
TongLee Chung*, Bin Xu*, Peng Zhang*, Yuanhua Tan**, Ping Zhu**, \\ Adeli $•$ Wubulihasimu*** \\ Knowledge Engineering Group, Department of Computer Science and Tecnology, Tsinghua \\ University, Beijing, China*, Hongyou Software Co Ltd**, PetroChina Urumqi Petrochemical \\ Company*** \\ tongleechung86@gmail.com, xubinetsinghua.edu.cn, \\ zpjumper@gmail.com, \{tanyh66,hy-zhp, aslws1\}@petrochina.com.cn,
}

\begin{abstract}
City Ontology plays an important role in smart city management for data integration, reasoning decision support etc. With these managerial domain knowledge scattered among a large number of experts, researchers face a huge challenge constructing a complete ontology for city management. This paper presents a simple yet efficient method for non-computer science experts to construct an ontology. We use a middle part that acts as a transition layer called activity model which is later merged into the city managerial ontology. We prove the effectiveness of this method by constructing a managerial ontology for two departments in Karamay's Smart City Program.
\end{abstract}

Keywords: Ontology, city management, smart city, managerial activity model

\section{Introduction}

An ontology is a model of a particular domain, built for a particular purpose [1]. Domain ontologies capture knowledge of one particular domain and are usually constructed manually [1]. OWL ${ }^{1}$ language is standardize by the World Wide Web Con-

1 http://www.w3.org/TR/owl-features/ 
sortium (W3C) Web Ontology Working Group for constructing ontology and is widely used by researchers and developers. A city managerial ontology describes managerial activities and processes in a city that can be used for data integration from different database, reasoning to fill in missing links and decision support.

Yet information for constructing a city managerial ontology is scattered among many experts, mostly not from computer science background. City management is a huge domain with many different fields and experts are those who have many years of experience [2]. These experts would appreciate that their expertise be stored as ontology instead of being preserved in books. The biggest challenge faced in ontology construction is to equipped domain experts with the right tool. It would be naive idea to have experts learn an ontology language and make them work together to construct a complete city ontology.

This paper puts forward a method for experts to construct a managerial ontology that doesn't request prerequisites of ontology language or knowledge. We first have expert to model their managerial knowledge as processes and activity which is used as a transition model. The managerial activity model is then transformed into an OWL form ontology where duplicate concepts are later merged.

This method is tested by constructing the ontology for Karamay's Urban Management department's management activities and process and oil exploration activities. The ontology is reasonable and rather complete that describes activates and procedures of their duty in city management.

The contributions of this paper are as followed:

- Provide experts a tool to construct ontology automatically from managerial activity model. Instead of having experts work in a field of ontology which they are not familiar with, let them model the process of managerial work in a way they are more comfortable with. This method will save developers and experts time and money constructing ontologies. This method will also reduce the communication barriers between domain experts and developers.

- Put forward an effective activity model for describing city managerial process. The managerial activity model is able to describe workflow of city management. This model gives experts abilities to describe their work in a form they are familiar with. And this model is also easy for developers to understand.

The rest of the paper is organized as follows: In section 2, we will present the managerial activity model. In section 3 , we will discuss the transformation process of 
managerial activity model to ontology and two success case using our method. In section 4 , we will briefly look at related works. And finally, we will conclude our paper in section 5 .

\section{Managerial Activity Model to Ontology}

Before going into details, we will first describe city managerial procedures and city managerial activities and the importance of ontology in city management. Then we will define some rules and regulations for modeling city managerial activity which makes transformation process possible.

\section{City Management and Ontology}

By city management, we mean managing functional departments of city government like police department, fire department etc. In a city, managing all these department efficiently requires integrating different databases and optimizing activities and etc. This is a difficult task and requires an understanding of all domain knowledge, a retentive memory and an agile logic in one person which is almost impossible.

An ontology is a model of a particular domain, built for a particular purpose [1]. It is handy to construct an ontology to include all procedure and activities in city management. A complete ontology can be useful in many areas. Data integration can be done with the help of ontology. Activity arrangement can be better optimized with the help of ontology. Reasoning and inference can be done on ontology for decision support. A huge challenge is how to construct such an ontology. City managing experts are often those who have been in the field for many years and only process a small portion of knowledge in city management. Usually, these people do not have a strong computer science background. How to get all these knowledge together and construct a complete ontology is a real big challenge.

\section{Management Activity Model}

A managerial activity model is a model that describes the workflow of an activity. We were greatly influenced by workflow business models [3,4] and workflow graph[5]. Experts in city management are more confident about their workflow than 
ontology, so it would be a good idea to have experts work on their comfort zone. There are a few requirement for the activity model that allows the model be transformed into an ontology directly.

Table 1. Constructing managerial activity model using Excel

\begin{tabular}{|c|c|c|c|}
\hline Identifier & Term & Category & Type \\
\hline $\mathrm{J}$ & Urban Management & domain & \\
\hline J. 3 & City appearance & function & \\
\hline J. 3.8 & Street standardize inspection & process & \\
\hline J. 3.8 .1 & Beforehand meeting & $\underline{\text { activity }}$ & \\
\hline J. 3.8.1.1 & Meeting date & Attribute & date \\
\hline J. 3.8.1.2 & Meeting room & Material & infrastructure \\
\hline J. 3.8.1.2.1 & Address & Attribute & string \\
\hline J. 3.8.1.3 & Chief inspector & Person & operator \\
\hline J. 3.8.1.3.1 & Name & Attribute & string \\
\hline J. 3. 8.1. 4 & Inspector & Person & participant \\
\hline J. 3.8.1.4. 1 & Name & Attribute & string \\
\hline J.3.8.1.5 & Meeting minutes for Inspection & Material & document \\
\hline J. 3.8.1.5.1 & Meeting date & Attribute & date \\
\hline J.3.8.1.5.2 & Meeting room & Material & infrastructure \\
\hline J.3.8.1.5.2.1 & Address & Attribute & string \\
\hline J.3.8.1.5.3 & Chairman & Person & operator \\
\hline J.3.8.1.5.3.1 & Name & Attribute & string \\
\hline J. 3.8.1.5.4 & Inspector & Person & participant \\
\hline J. 3.8.1.5.4.1 & Name & Attribute & string \\
\hline J. 3.9 .1 & On-street inspection & activity & \\
\hline J.3.9.1.1 & inspection date & Attribute & date \\
\hline J. 3.9.1.2 & inspection duration & Attribute & digit \\
\hline J. 3.9 .1 .3 & Chief inspector & Person & operator \\
\hline J. 3.9.1.3.1 & Name & Attribute & string \\
\hline J. 3.9.1. 4 & Inspector & Person & participant \\
\hline J. 3.9.1.4. 1 & Name & Attribute & string \\
\hline J. 3.9.1.5 & Vehicle & Material & device \\
\hline J.3.9.1.5.1 & Plate number & Attribute & digit \\
\hline J. 3.9.1. 6 & Two way radio & Material & device \\
\hline J. 3.9.1.6. 1 & Radio number & Attribute & digit \\
\hline J.3.9.1.12 & Inspection report & Material & document \\
\hline J. 3.9.1.12.1 & inspection date & Attribute & date \\
\hline J.3.9.1.12.1.1 & inspection duration & Attribute & digit \\
\hline J. 3.9.1.12.1.2 & Chief inspector & Person & operator \\
\hline J.3.9.1.12.1.3 & Name & Attribute & string \\
\hline J. 3.9.1.12.1.4 & Inspector & Person & participant \\
\hline J.3.9.1.12.1.5 & Name & Attribute & string \\
\hline J. 3.9.1.12.1.6 & Vehicle & Material & device \\
\hline J. 3.9.1.12.2 & Plate number & Attribute & digit \\
\hline J.3.9.1.12.2.1 & Two way radio & Material & device \\
\hline J. 3.9.2.1 & Radio number & Attribute & digit \\
\hline
\end{tabular}

The basic unit of the model is activity and cannot be divided. Activity consists of people, venue, time, objects etc. This is a reasonable and complete form to describe activities in city management and can be easily understood. A procedure is a set of activities in a sequential order. A function is a set of procedure in a sequential order. And the certain domain consists of a series of functions. This makes up the upper layer of city management in a hierarchical form. The bottom layer consists of objects and attributes. Objects are physical things that were involved in an activity. Objects are separated into two categories: persons and non-person. Person describes the people 
that were involved in the activity. We define two types of people in an activity: operator and participant. Operator by definitions is the person operating or the person in charge and participant is people that the activity involves. We use material to define other non-person objects. Material type includes infrastructure, device, document and etc. Attribute is used to enrich descriptions of objects and activities. Types of attributes include date, string, digit etc. The output of management activities are usually a report that concludes the activity.

The model contains four columns: Identifiers, terms, category and type. Identifier is used to indexing and showing hierarchical relation. Term is basically the name of the concept. Category is used to determine which category the item falls into. And the type defines the type of the item. We have already define category and type in the above section.

This model can be understood by experts easily and matches the logic they have in mind for managing a city in their own field. This model allows experts to focus only on their expert field and ignore ambiguity and communication problem they will have to face when working together. The tools used to construct this model is as simple as Excel. This makes expert's works simple and focused. This model doesn't require experts to have knowledge about ontology or computer science. It lets them better express their knowledge as it should be. Table 1 is a sample from experts in Urban Management Department. The underlined item represents an activity.

\section{Constructing Ontology from Activity Model.}

Design of the activity model is intended to construct a city managerial ontology without having experts look at the ontology. The design itself consist of an ontology that we consider the base of the city ontology. The requirements of activity model is based on this base model.

The activity model can be easily constructed into a tree using the identifiers. Each level has a concept in the base ontology that is the superClass. The tree is divided into two part: the upper level and the lower level. The upper level is the hierarchy tree of activity. We use the relation "hasFunction", "hasProcess", "hasActivity" to denote the corresponding relations. This part of the tree is preserved as to represent the flow of work. We use two relations to represent workflow: "first" and "next". In the upper level, the "first" relation indicates that it is the first child of all siblings and "next" relation to indicate that it is the next in the set of children. We use type column to 
define predicates between two concepts. Next we build sub-predicate relation for predicate linking to base ontology.

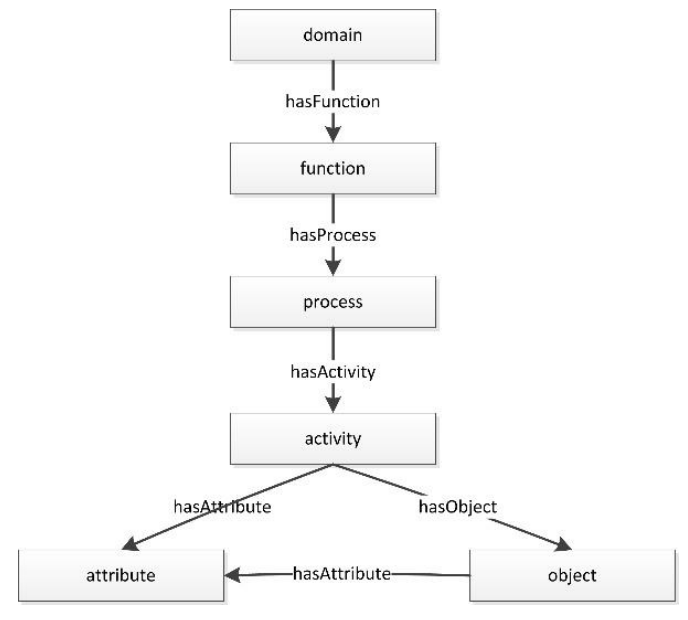

Fig. 1. Base ontology

The lower level of the tree contains objects and attributes, which is the part we have to merge same concepts. There are two strategies we use for merging the same concept. The basic strategy is using the term of the item. If it has the same term then we consider that they are the same concept. Strategy two is more complicated. We first compare the cosine similarity of the term, if it exceeds a threshold then we compare the semantic similarity based on linked node counting. Similarity is calculated as the quotient of same concept linked over the total number of linked concepts. We add a normalizing factor to smoothen the results.

But since this is a strict ontology, we won't have machine do all the job, we will enquire expert confirmation before we merge concepts. That will require experts who define the term to confirm whether the two concepts should be merged. We will lower the load for them by finding possible concepts to merge. And finally, we output this ontology in OWL format. Figure 2 show a graph ontology of the city managerial model given in Table 1. We omit the subPredicate relation from the graph to make it cleaner.

We were very fortunate to be able to test our method in Karamay. Karamay is an oil city with success in digitization. We tested our method in two departments: Oil Exploration Department and Urban Management Department. We asked experts in 
these field to model their management process with the management activity model. They easily understood the requirements and finished modelling rather quickly. We transformed these model into one ontology and had these experts look at the ontology. They considered it to be complete and compatible. The ontology is outputted in OWL format and will be used later in data integration and decision support engine.

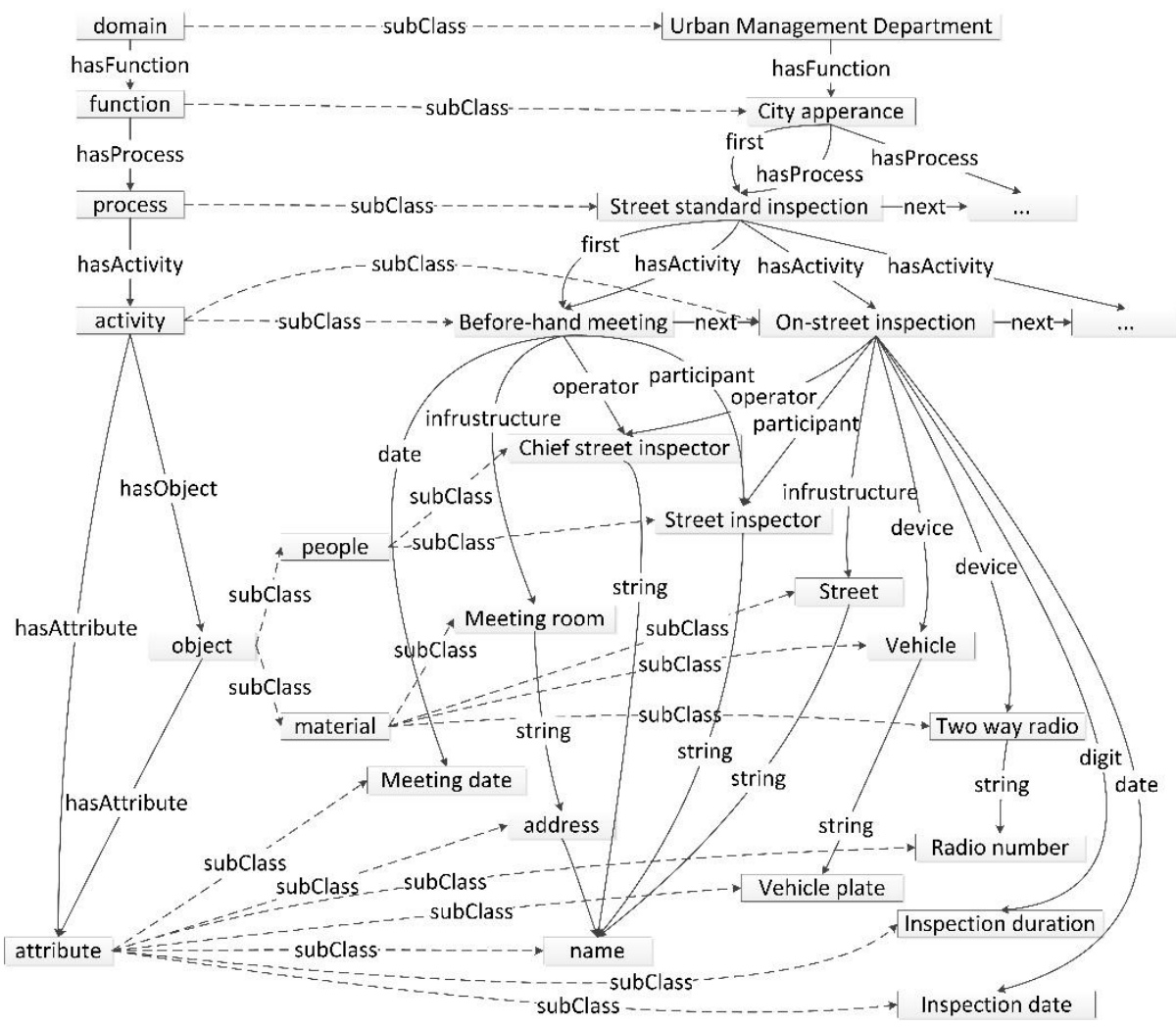

Fig. 2. City management ontology from managerial activity model

Table 2. Stats of ontology construction

\begin{tabular}{lccc}
\hline Column1 & \# of activity model & \# of concpets & \multicolumn{2}{c}{ \# of links } \\
\hline Oil Exploration Department & 23 & 9890 & 40572 \\
Urban Management Department & 15 & 5200 & 24345 \\
total & 38 & 15090 & 64917 \\
\hline
\end{tabular}




\section{$4 \quad$ Related work}

Most domain experts are not from computer science field, most of them do not have the understanding to computer science technologies. We wish to give them the right tools to construct an ontology. Researchers have come up with many tools and methods to construct ontology. Jena is a java API that has full support of ontology construction and common task to perform [6]. Protégé Ontology Editor is an ontology editor and knowledge base framework that is easy to construct an ontology [7]. Bouillet propose a method where several experts can construct an ontology in a collaborative manner [8]. But these tools and methods are not the right tools for experts to construct ontology. Experts will have to construct the ontology manually, sometimes without even knowing what the ontology looks like thus increasing the burden of experts. Also, experts will have to collaborate a lot making some of them lose focus on the areas they are skilled at. Our method allows expert describe workflow and activities instead of directly constructing ontology. Our method lets expert works collaborative in a manner that they only focus on their own field.

There are many research on smart cities. Beirao construct a street description for a city ontology [9]. Jun Zhai propose a system architecture of integrated information platform for digital city based on ontology [10]. There has been a lot of breakthrough in the field of smart city with ontology, but with very little consideration of providing an efficient way to construct ontology.

\section{$5 \quad$ Conclusion and Future Work}

With the rapid development and digitalization of cities, there is an urgent need to construct an ontology for developing intelligent system for smart city support. But experts face the problem of learning ontology technology, computer science technology and cooperating with many other experts. In this paper, we propose a method that allows non-computer science experts to efficiently preserve their expert knowledge as ontology. We introduce a middle transition model called management activity model that is fairly easy for experts to model their expert field and can be directly converted to ontology requiring only a small process for experts.

As future work, we will try to extend the method to easily construct hierarchy in objects and attributes, for example, name is a super-class of street name and people name. We would like to find missing links within the ontology, for example, street 
inspector include chief street inspector. We will also have more experts to model the whole domain of city ontology of Karamay. Another important part of future is to select vocabulary so that our work can be reused properly.

Acknowledgments: This work is supported by the China National High-Tech Project (863) under grants No SS2013AA010307.

\section{Reference}

1. Grigoris Antoniou, Paul Grouth, Frank van Harmelen, Rinke Hoekstra. A Sementic Web Primer $3^{\text {rd }}$ Edition. 2012. Chapter 7. Pages 193-213.

2. Amy K. Donahue, Sally C. Selden, and Patricia W. Ingraham. Measring Government Management Capacity: A Comparative Analysis of City Human Resources Management Systems, Journal of Public Administration Research and Theory (2000)10 (2). Pages $381-412$.

3. Myers, K. \& berry, P. 1998, Workshop Management System: An AI Perspective. AIC-SRI report 1-34.

4. Michael Morries, Minet Schindehutte, Jeffery Allen. The entrepreneur's business model: towards a unified perspective. Journal of Business Research (2005). 726-735

5. Johann Eder, Wolfgang Gruber, Horst Pichler. Transforming Workflow Graphs. INTEROP-ESA, 2005, Genf, Switzerland.

6. http://jena.apache.org/documentation/ontology/

7. http://protege.stanford.edu/

8. Eric Bouillet, Mark Feblowitz, Zhen Liu, Anand Ranganathan, Anton Riabov. A Knowledge Engineering and Planning Framework based on OWL ontologies. Association for the Advancement of Artificial Intelligence.

9. Jose Beirao, Nuno Monttenegro, Jorge Gil. The city as a street system: A street description for a city ontology. SIGraDi $2009 \mathrm{sp}$.

10. Jun Zhai, Jiatao Jiang, Yi yu, Jianfeng Li. Ontology-based Integrated Information Platform for Digital City. WiCOM 2008, $4^{\text {th }}$ International Conference. 